\title{
PHILOSOPHICAL
}

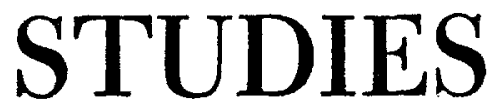

Edited by WILFRID SELLARS and HERBERT FEIGL with the advice and assistance of PAUL MEEHL, JOHN HOSPERS, MAY BRODBECK

VOLUME XVII

Contents October 1966

NUMBER 5

Do Relations Individuate? by J. W. Meiland, UNIVERSITY OF MICHIGAN

Scriven on Human Unpredictability by David K. Lewis and

Jane Shelby Richardson, HARVARD UNIVERSITY

Assertion and Belief by Charles Sayward, unIVERSITY OF NEBRASKA

\section{Do Relations Individuate?}

\author{
by J. W. MEILAND
}

UNIVERSITY OF MICHIGAN

WHAT is it that renders two exactly similar material objects numerically different from one another? One possible answer to this question is that the two objects have different relational properties or stand in different relations. They stand in different spatial relations to some other material objects and, perhaps, to one another. For example, one object $X$ may be to the left of the other, $Y$ (this relation hereafter being called "Relation $L$ "), while $Y$ is to the right of $X$. E. B. Allaire rejects this answer on the grounds that $X$ and $Y$ first must be numerically different before they can stand in Relation $L$ : "Relations-I'll stick with spatial ones-presuppose numerical difference; they do not account for it. The thisness and the thatness of things is presupposed in saying that the one is to the left of the other."1 Allaire's own answer to the question stated above is that each of the two numerically different material objects contains a different "bare particular" (a particular of this sort being called "bare" because it itself has no properties): "Bare particulars are, 
therefore, the entities in things accounting for the numerical difference of things." I wish to show that the theory of bare particulars is highly implausible and that relations do individuate material objects.

\section{I}

What is Allaire's argument for the claim that "relations presuppose numerical difference?" He says that if this were not so, "then in at least some cases we would be forced to say what we all know to.be false; namely, that the same thing is to the left of itself."3 But perhaps saying that the same thing is to the left of itself is false just because Relation $L$ does individuatethat is, just because if $X$ and $Y$ stand in Relation $L$ to one another, then it is logically impossible for $X$ and $Y$ to be numerically identical. Perhaps that $X$ and $Y$ stand in Relation $L$ is a logically sufficient condition of the numerical difference of $X$ and $Y$. If so, then standing in that relation would by itself guarantee that the terms of the relation are numerically different; and thus bare particulars would not be required to account for this difference.

Allaire denies that standing in Relation $L$ is a logically sufficient condition of numerical difference. He admits that Relation $L$ "cannot be exemplified by one thing." But he says: "That $L$ is asymmetrical is factual, not logical." 4 Since asymmetry implies irreflexivity, Allaire is claiming that it is a factual matter, not a logical truth, that Relation $L$ is irreflexive. He does not give any argument for this claim. But this claim is crucial to the plausibility of this theory of bare particulars, as I now wish to show.

If it is merely a factual matter that Relation $L$ cannot be exemplified by one thing, then it is logically possible that it be exemplified by one thing. Let us suppose that we are observing a case of what we would describe as "one material object $\mathrm{X}$ being to the left of another, $\mathrm{Y}$." Since on the theory of bare particulars, objects contain bare particulars, Allaire would say that if this is in fact a case involving two different objects, then there is one bare particular in the location occupied by $X$ and a different bare particular in the location occupied by $Y$. But, if Allaire is correct about Relation $L$, it is logically possible that this case is not a case involving two different objects, $X$ and $Y$, even though in every observable respect this case is just like a case involving two different objects. That is, it is logically possible that the numerically same bare particular occupies two different locations (those which, as we say, are occupied by $X$ and $Y$ ). So in claiming that the irrefiexivity of Relation $L$ is factual, not logical, Allaire is claiming that the numerically same bare particular can occupy two different locations. But there is no way to determine, in a case of the sort described above, whether the two locations are occupied by different bare particulars or by the same bare particular, for what is observed is the same in either case. Hence, if Allaire is correct, there 
is no way to determine whether such a case is a case of numerical difference or of numerical identity. That $X$ and $Y$ occupy different locations does not settle the matter, if Allaire is correct, since those different locations may be occupied by the same bare particular.

But then what basis could anyone have for asserting that in a case of the sort described above, $X$ and $Y$ are numerically different or that they are numerically the same? One could not even have an inductive basis for such assertions. For to have an inductive basis for this, one would have to have found that in some previous cases of objects that occupy different locations, those objects were numerically different (or identical). But in order to acquire such inductive evidence, we would first have to possess a way of determining whether a given case is a case of numerical identity or of numerical difference.

Since there could be no basis for saying, with certainty or with probability, that two exactly similar material objects are numerically identical or numerically different, one could never know, in a given case, how many bare particulars are present. So the theory of bare particulars provides an account of the numerical differences of things at the price of making it impossible for us to determine whether or not a given case is a case of numerical difference. Thus, if the theory of bare particulars is correct, it can never be employed in a particular case. The only reason that Allaire gives for supposing that there are bare particulars is that they are required to "account for the numerical difference of things." But if the theory of bare particulars is correct, then no cases could be known to exist for which it was needed to account. For although bare particulars are supposed to explain numerical difference rather than provide a criterion for detecting numerical difference, the existence of bare particulars would seem to eliminate the possibility of having any criterion for detecting numerical difference in cases of two bodies that have exactly the same properties (other than spatial properties).

It is true that one could still say: "If this particular case is a case of numerical difference, then it is a case involving two different bare particulars." But is to say that two different bare particulars are present in such a case to say anything more than that it is a case of numerical difference? And if it is not to say anything more than this, then in what sense does saying that different bare particulars are present account for numerical difference?

Could Allaire say that the irreflexivity of Relation $L$ is logical rather than factual? To do so would meet the objections above. But to do so would also allow relations to individuate. For then standing in Relation $L$ would by itself, regardless of the existence or nonexistence of bare particulars, guarantee numerical difference. Objects would be numerically different solely in virtue of standing in Relation $L$. And then there would be no need to postulate the 
existence of bare particulars. Numerical difference could be accounted for by citing the relations in which the objects in question stand.

II

There are two sorts of situations which involve numerical identity or difference. The first concerns objects existing at a given moment; the second concerns objects existing at different moments. As a situation of the first sort, let us suppose that two exactly similar objects $X$ and $Y$ coexist but occupy different locations. If it is only factually the case, rather than logically necessary, that $X$ and $Y$ are numerically different, then it is logically possible that exactly similar objects which occupy different locations are numerically identical. But then what would it mean to say of such objects that they are numerically identical? If we do not employ a theory of substance as substratum or a theory of bare particulars, there seems to be nothing that could be meant by saying that they are numerically identical; if it is only factually the case that $X$ and $Y$ are numerically identical, then a situation that is exactly the same as the described situation in every observable respect could be a situation involving numerical difference of objects. So unless we say that ascriptions of numerical identity and difference in situations of this first sort are about something unobservable (a substratum or bare particulars), we must say either (i) that ascription of numerical identity or difference in situations of this first sort has no meaning, or else (ii) that what it means to say that two exactly similar objects are numerically different is that they occupy different locations. That is, in order to provide a meaning for such ascriptions without referring to something unobservable, we must deny that it is a merely factual matter that objects occupying different locations are numerically different. We must say that "occupying different locations" is what the expression 'numerically different' means in such a situation. Only in this way will it not be a merely factual matter that objects occupying different locations are numerically different. And hence only in this way will the expression 'numerically different' have a meaning in the first type of situation without invoking something unobservable.

In every situation of the first sort-that is, situations involving coexisting material bodies-two such bodies are numerically different if and only if (or when and only when) they occupy different locations. The meaning of the expression 'numerically different' is "occupying different locations." But the following objection might be raised to this position:

This position on the meaning of 'numerically different' is satisfactory only if we consider only the first sort of case. Let us now consider situations in which the two entities in question do not coexist. We often say that what I am now observing (call this " $X$ ") is numerically different from what I 
previously observed (call this "Y"). Yet what I now observe can be both exactly similar to and in the same location as what I previously observed, even though the two things are numerically different. Therefore the expression ' $\mathrm{X}$ is numerically different from $\mathrm{Y}$ ' cannot mean " $\mathrm{X}$ and $\mathrm{Y}$ occupy different locations," unless the expression "numerically different' is equivocal, that is, unless it has one meaning in the first sort of situation and a different meaning in the second sort of situation.

What this objection shows is that we must add to the expression which renders the meaning of 'numerically different': "Two objects are numerically different (a) if they coexist, then when and only when they occupy different locations, or (b) in case they in fact never coexist, if and only if they would have occupied different locations had they coexisted." But to make the addition represented by part (b) is only to add further specification concerning the locations of bodies. It is not to mention something unobservable which is said to be contained within material objects. It seems, then, that a substratum or bare particulars need not be postulated if the expression "numerical difference' has the meaning suggested above. But if it has the suggested meaning, then relations do individuate. For then $X$ and $Y$ are numerically different when and only when they have or would have had different locations, that is, when and only when they have or would have had different spatial relations to other bodies.

Received April 19, 1965

NOTES

${ }^{1}$ E. B. Allaire, "Another Look at Bare Particulars," Philosophical Studies, 16:19 (1965).

${ }^{a}$ Ibid., p. 18.

${ }^{3}$ Ibid., p. 19.

${ }^{4}$ Ibid.

\section{Scriven on Human Unpredictability}

by DAVID K. LEWIS and JANE SHELBY RICHARDSON

HARVARD UNIVERSITY

IN HIs paper "An Essential Unpredictability in Human Behavior,"1 Michael Scriven offers an argument intended to show that it is impossible in principle to predict what a person (or indeed a suitable robot) will do in a certain 